\title{
Sorting Out the JUNQ: the Spatial Nature of Protein Quality Control
}

\author{
Emily Sontag ${ }^{1}$, Jian-Hua Chen ${ }^{2}$, Gerry McDermott ${ }^{2}$, Dan Gestaut ${ }^{1}$, Carolyn Larabell ${ }^{2,3}$ and Judith
} Frydman $^{1}$

1. Department of Biology, Stanford University, Stanford, CA 94305, USA

2. Department of Anatomy, School of Medicine, University of California San Francisco, San Francisco, CA 94158, USA

3. Molecular Biophysics and Integrated Bioimaging Division, Lawrence Berkeley National Laboratory, Berkeley, CA 94720, USA

A healthy proteome is essential for survival, and cells have elaborate protein quality control (PQC) systems to maintain protein homeostasis, or proteostasis [1,2]. PQC systems are comprised of molecular chaperones which identify and bind misfolded proteins then target them for clearance by proteolytic pathways such as the proteasome or autophagy [1]. Increasingly, defects in PQC are linked to human diseases such as Alzheimer's, Parkinson's, cancer and even aging [1-2]. One commonality among many of these diseases is the presence of intra- and extra-cellular inclusions containing misfolded proteins, which may result from a breakdown in PQC mechanisms leading to terminal sequestration of misfolded, toxic proteins. This demonstrates a critical need to better understand the complex PQC machinery as well as the mechanisms of inclusion formation and clearance. Misfolded proteins are actively sequestered into a number of distinct PQC compartments that are conserved from yeast to mammals [1]. The juxtanuclear quality-control compartment (JUNQ), which forms when the proteasome is impaired, contains soluble misfolded proteins that can be refolded or cleared [3]. A recent study proposed that the JUNQ actually resides inside the nucleus and should be renamed to INQ [4].

In order to examine the exact location of the perinuclear compartments, we utilized a mutant Luciferase model protein fused to either a nuclear localization signal (NLS) or a nuclear export signal (NES). This mutant Luciferase misfolds upon heat shock and gets sorted to different PQC compartments. To confirm that the resulting compartments are, in fact, in the nucleus and cytoplasm, we performed Structured Illumination Microscopy. Yeast were immune-stained for GFP positive inclusions, anti-Nsp1 for nuclear envelope visualization and Hoechst for DNA as a visual reference. The nuclear Luciferase is indeed confined to a nuclear PQC compartment - likely the INQ, whereas the cytoplasmic Luciferase is sequestered in a cytoplasmic, juxtanuclear compartment - likely the JUNQ (Figure 1). As this technique required a substantial amount of processing that could alter the samples, and we couldn't determine if the juxtanuclear compartment was in a nuclear invagination, we turned to soft X-ray tomography (sXRT) to visualize the nuclear and cytoplasmic compartments. We co-expressed the NLS and NESLuciferase constructs in the same cells and the samples were imaged using a transmission soft X-ray microscope, operated by the National Center for X-ray Tomography (NCXT), at the Advanced Light Source of Lawrence Berkeley National Laboratory and reconstructed according to previously published protocols. Again, we see the nuclear and cytoplasmic Luciferase confined to separate PQC compartments in the nucleus and cytoplasm - likely the INQ and the JUNQ respectively (Figure 2).

In summary, misfolded nuclear and cytoplasmic proteins are quarantined to separate PQC compartments depending upon their location. By combining multiple imaging techniques, we can visualize the dynamic sorting of misfolded proteins to their respective PQC compartments and pinpoint the location of the compartments by super-resolution microscopy and sXRT. Future studies will utilize sXRT to 
examine the internal structures within these compartments. As they have only been studied by fluorescence imaging previously, using sXRT, we can determine any differences in density inside the compartments and between different types of compartments. This will provide enormous insight into the formation of the compartments and begin to lend insight into possible functions of the compartments as well. This knowledge is critical to understanding the etiology of various misfolded protein diseases and for developing novel and potent therapeutic strategies as well [5].

\section{References:}

[1] EM Sontag, WI Vonk and J Frydman, Curr Opin Cell Biol 26 (2014), p. 139-46.

[2] J Tyedmers, A Mogk and B Bukau, Nat Rev Mol Cell Biol 11 (2010), p. 777-88.

[3] D Kaganovich, R Kopito and J Frydman, Nature 454 (2008), p. 1088-95.

[4] SB Miller, A Mogk and B Bukau, J Mol Biol 427 (2015), p. 1564-74.

[5] We acknowledge funding from the NIH and a Senior Scholar Award from the Ellison Foundation to JF. ES was supported by a postdoctoral fellowship from NIH (F32NS086253). The National Center for X-ray Tomography is supported by NIH (P41GM103445) and DOE's Office of Biological and Environmental Research (DE-AC02-5CH11231)

A

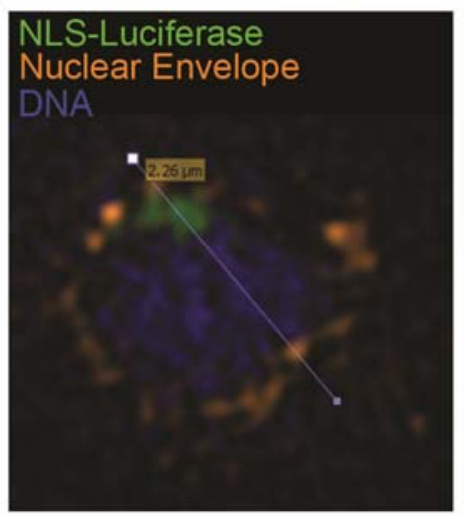

$\mathrm{B}$

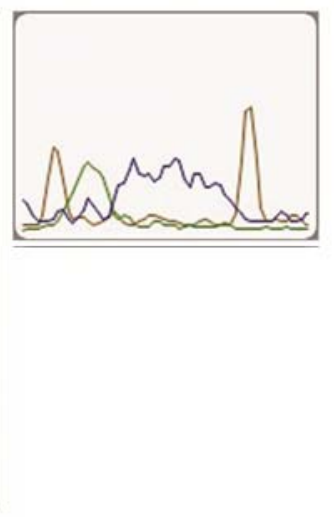

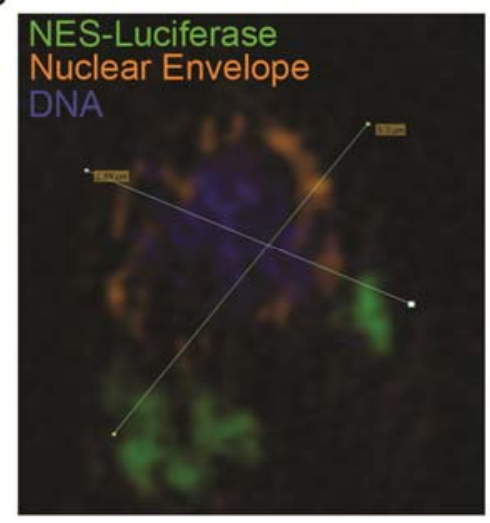

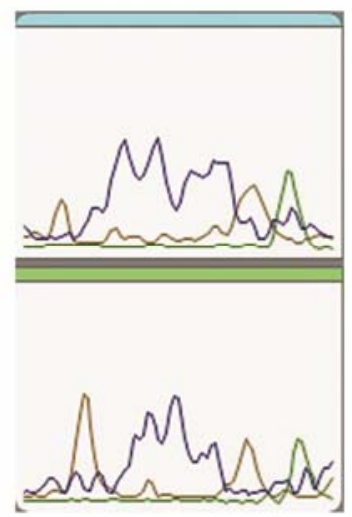

Figure 1. Structured Illumination Microscopy of (A) nuclear misfolded Luciferase including histogram and (B) cytoplasmic misfolded Luciferase including histogram.
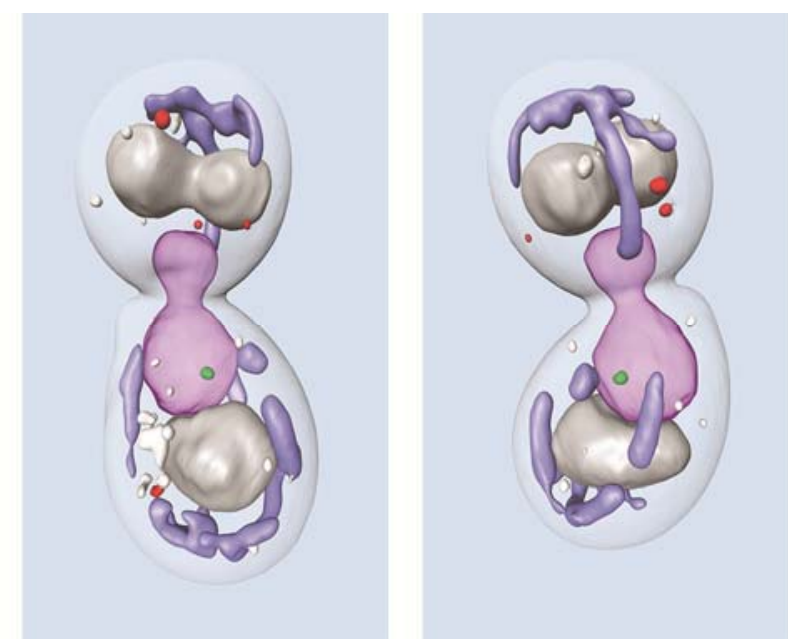

\author{
NLS-Luciferase \\ NES-Luciferase \\ Nucleus \\ Mitochondria \\ Vacuoles \\ Lipid droplets - white
}

Figure 2. Soft X-ray Tomography of yeast cells co-expressing nuclear and cytoplasmic misfolded Luciferase. Two views of the same cells are shown. 